\title{
CAMBIOS EN LA FUNCIÓN ENDOTELIAL VASCULAR DE PACIENTES CON ARTRITIS REUMATOIDEA EN TRATAMIENTO
}

Publicado:

16 de diciembre, 2020 


\section{Autores}

ZACARIAZ Hereter, Johana MD

Sección de Reumatología, Servicio de Medicina Interna, Hospital Italiano de Buenos Aires

LANCIONI, Eliana MD

Sección de Reumatología, Servicio de Medicina Interna, Hospital Italiano de Buenos Aires

SCHNEEBERGER, Emilce E MD Instituto de Rehabilitación Psicofísica, Buenos Aires

CAZENAVE, Thomas MD

Instituto de Rehabilitación Psicofísica, Buenos Aires

APARICIO, Lucas S MD

Sección de Hipertensión, Servicio de Medicina Interna, Hospital Italiano de Buenos Aires
NORSCINI, Jorge MD

Servicio de Neurología, Hospital Italiano de Buenos Aires

ROSA, Javier MD

Sección de Reumatología, Servicio de Medicina Interna, Hospital Italiano de Buenos Aires

CITERA, Gustavo MD

Instituto de Rehabilitación Psicofísica, Buenos Aires

SORIANO, Enrique R. MD, MSC

Sección de Reumatología, Servicio de Medicina Interna, Hospital Italiano de Buenos Aires 
ARTículo CiENTífico

VASCULAR ENDOTHELIAL

FUNCTION CHANGES DURING

TREATMENT IN PATIENTS WITH

RHEUMATOID ARTHRITIS 


\section{en Español}

\section{Introducción}

La velocidad de onda de pulso carótida-femoral (VOP) y el espesor de la íntima media de la Arteria Carótida Común (EIM-ACC), son marcadores indirectos de aterosclerosis. Los fármacos antirreumáticos modificadores de la actividad (FARME), controlando la inflamación, podrían influir en el desarrollo de aterosclerosis. El objetivo de nuestro estudio fue evaluar el efecto a corto plazo de los FARME, sobre los marcadores surrogados de aterosclerosis en pacientes con AR.

\section{Materiales y métodos}

Estudio prospectivo, incluyó pacientes consecutivos con AR (criterios clasificatorios ACR 1987), en quienes se prescribió inicio o cambio de FARME, divididos en 3 grupos de tratamiento: abatacept [FARME-b (Aba)], inhibidor de TNF-a [FARME-b (TNFi)], y FARME convencional (FARME-C). Fueron excluidos pacientes con antecedentes de enfermedad cardiovascular. La evaluación clínica y cardiovascular fue realizada al inicio, y luego de 12 meses. Las mediciones de
EIM-ACC y VOP se realizaron usando ultrasonido de alta resolución, y sonda de tonometría manual

\section{Resultados}

Fueron incluidos 64 pacientes con AR, 89,3\% eran mujeres, edad media: 56.7 (DE: 12.1) años. Se perdieron durante el seguimiento 8 pacientes. Se incluyeron en el análisis final 56 pacientes [FARME-b (Aba): 15, FARME-b (TNFi): 18 y FARME-C: 23]. Al inicio del estudio, 24 pacientes (43\%) tenían aterosclerosis. En el seguimiento 4 nuevos pacientes desarrollaron aterosclerosis [FARME-b (Aba): grupo: 1, grupo FARME-b (TNFi): 2 y grupo FARME-C: 1].

\section{Conclusión}

Después de un año de tratamiento, no se encontraron cambios significativos en los marcadores sustitutos de aterosclerosis [media $4,7$ (DE: 1,3) vs 4 (DE: 1, 2); $p=0,0425]$. 


\section{en Inglés}

\section{Introduction}

Carotid-femoral pulse wave velocity (VOP) and thickness of intima media of Common Carotid Artery (EIM-ACC), are atherosclerosis indirect markers. Disease-modifying antirheumatic drugs (DMARDs), could influence the atherosclerosis development, by controlling inflammation. The objective of our study was to evaluate the short-term effect of DMARD treatment on surrogate markers of atherosclerosis in patients with RA.

\section{Material and methods}

This prospective study included consecutive patients with RA (ACR 1987 classification criteria) who were prescribed to start or change DMARD, divided into 3 treatment groups: abatacept [bDMARD (Aba)], TNF-a inhibitor [bDMARD (TNFi)], and conventional DMARD (CDMARD). Patients with cardiovascular disease history, were excluded. Rheumatological clinical assessment and cardiovascular risk were performed at baseline, and after 12 months. EIM-ACC and VOP measurements were performed using high-resolution ultrasound and manual tonometry probe.

\section{Results}

We included 64 patients with RA, $89.3 \%$ were female, mean age: 56.7 (SD: 12.1) years. Eight patients were lost to follow up, the remaining 56 patients were included in the final analysis [bDMARD (Aba): 15, bDMARD (TNFi): 18 and CDMARD: 23]. At baseline 24 patients (43\%) had atherosclerosis by ultrasound. At follow up 4 new patients fulfilled atherosclerosis criteria [bDMARD (Aba): group: 1, bDMARD (TNFi) group: 2, and CDMARD group: 1].

\section{Conclusion}

After one year of treatment, no significant changes in surrogate markers of atherosclerosis where found [mean 4.7 (SD: 1.3) vs 4 (SD: 1.2); $p=0.0425]$. 


\section{en Portugués}

\section{Introdução}

A velocidade da onda de pulso carotídeo-femoral (VOP) e a espessura da média íntima da Artéria Carótida Comum (EIM-ACC), são marcadores indiretos de aterosclerose. As drogas antirreumáticos modificadores da doença (DMARD) podem influenciar o desenvolvimento da aterosclerose, controlando a inflamação. 0 objetivo de nosso estudo foi avaliar o efeito de curto prazo do DMARD em marcadores substitutos de aterosclerose em pacientes com AR.

\section{Materiais e métodos}

Este estudo prospectivo incluiu pacientes consecutivos com AR (critérios de classificação ACR 1987) que foram prescritos para iniciar ou alterar DMARD, dividido em 3 grupos de tratamento: abatacept [DMARD-b (Aba), inibidor de TNF-a [DMARD-b (TNFi)], e DMARD convencional (DMARD-c). Pacientes com história de doença cardiovascular foram excluídos. A avaliação clínica reumatológica e o risco cardiovascular foram realizados no início do estudo e após 12 meses. EIM-ACC e VOP medições foram realizadas usando ultrassom de alta resolução e sonda de tonometria manual.

\section{Resultados}

Incluímos 64 pacientes com AR, 89,3\% eram mulheres, idade média: 56,7 (desvio padrão: 12,1) anos. Oito pacientes foram perdidos para acompanhamento, os restantes 56 pacientes foram incluídos na análise final [DMARD-b (Aba): 15, DMARD-b (TNFi):18 y DMARD-c: 23]. No início do estudo, 24 pacientes (43\%) tinham aterosclerose por ultrassom. No acompanhamento, 4 novos pacientes preencheram os critérios de aterosclerose [DMARD-b (Aba): grupo: 1, grupo DMARD-b (TNFi): 2 e grupo DMARD-c: 1].

\section{Conclusão}

Após um ano de tratamento, não foram encontradas alteraçoes significativas nos marcadores substitutos para a aterosclerose [média 4,7 (DP: 1,3) vs 4 (DP: 1, 2); $p=0,0425]$. 
Está demostrado que los pacientes con artritis reumatoide (AR) tienen una esperanza de vida reducida, y que entre el 35-50\% del exceso de mortalidad en estos pacientes es atribuible a enfermedades cardiovasculares (CVD) (1-5), con alto riesgo de aterosclerosis subclínica (6 y 7). Sin embargo, este exceso de mortalidad, no se explica por los factores de riesgo cardiovascular tradicionales (8-11). La disfunción endotelial, la rigidez arterial y las anomalías vasculares estructurales son probados marcadores surrogados de aterosclerosis prematura, potencialmente reversible y de eventos cardiovasculares (12-17). Estos marcadores podrían evaluarse midiendo la velocidad de la onda de pulso carótido-femoral (VOP) y el grosor íntima-media de la arteria carótida común (EIM-ACC), respectivamente. Los análisis de ondas de pulso (OPA) permiten estimar el índice de aumento central (IAC), una medida de la reflexión de la onda de pulso. IAc y OPA son indicadores de la rigidez arterial y tienen valor pronóstico en el desarrollo de enfermedades cardiovasculares (18-21). A pesar de la amplia variedad de estudios que informan los efectos cardiovasculares del tratamiento antirreumático, la identificación de estrategias de tratamiento que controlen tanto la actividad de la AR como la progresión de la enfermedad cardiovascular sigue siendo difícil de alcanzar (22-27). Si bien la información disponible sobre los efectos cardioprotectores de los FARME es cada vez mayor, esta literatura aún es escasa en la población de América Latina y existen pocos estudios con abatacept (28-30). El objetivo de nuestro estudio fue evaluar el efecto a corto plazo (12 meses) del tratamiento con FARME (incluyendo biológicos), en pacientes con AR.Está demostrado que los pacientes con artritis reumatoide (AR) tienen una esperanza de vida reducida, y que entre el 35-50\% del exceso de mortalidad en estos pacientes es atribuible a enfermedades cardiovasculares (CVD) (1-5), con alto riesgo de aterosclerosis subclínica (6 y 7). Sin embargo, este exceso de mortalidad, no se explica por los factores de riesgo cardiovascular tradicionales (8-11). La disfunción endotelial, la rigidez arterial y las anomalías vasculares estructurales son probados marcadores surrogados de aterosclerosis prematura, potencialmente reversible y de eventos cardiovasculares (12-17). Estos marcadores podrían evaluarse midiendo la velocidad de la onda de pulso carótido-femoral (VOP) y el grosor íntima-media de la arteria carótida común (EIM-ACC), respectivamente. Los análisis de ondas de pulso (OPA) permiten estimar el índice de aumento central (IAc), una medida de la reflexión de la onda de pulso. IAc y OPA son indicadores de la rigidez arterial y tienen valor pronóstico en el desarrollo de enfermedades cardiovasculares (18-21). A pesar de la amplia variedad de estudios que informan los efectos cardiovasculares del tratamiento antirreumático, la identificación de estrategias de tratamiento que controlen tanto la actividad de la AR como la progresión de la enfermedad cardiovascular sigue siendo difícil de alcanzar (22-27). Si bien la información disponible sobre los efectos cardioprotectores de los FARME es cada vez mayor, esta literatura aún es escasa en la población de América Latina y existen pocos estudios con abatacept (28-30). El objetivo de nuestro estudio fue evaluar el efecto a corto plazo (12 meses) del tratamiento con FARME (incluyendo biológicos), en pacientes con AR. 
Entre marzo de 2012 y febrero de 2014, se reclutaron 64 pacientes consecutivos vistos en la unidad de Reumatologia del Hospital Italiano de Buenos Aires, y el Instituto de Rehabilitación Psicofisica de Buenos Aires, Argentina, que cumplían los criterios de clasificación ACR de 1987 para AR (31-33) en quienes su reumatólogo tratante prescribió inicio o cambio de tratamiento con un nuevo FARME (incluyendo FARME biologico). Fueron clasificados en 3 grupos, pacientes que iniciarían tratamiento con FARME convencional (FARMEC), pacientes que iniciarían tratamiento con inhibidor del TNF [FARME-b (TNFi)], y aquellos que iniciarían tratamiento con abatacept [FARME-b (Aba)]. Al final del estudio, todos los pacientes permanecieron en el grupo en el cual iniciaron.

Los pacientes con antecedentes de enfermedad cardiovascular fueron excluidos del estudio.
Todas las mediciones se realizaron a los 0 y 12 meses. Un ecografista experimentado realizó todas las ecografías bidimensionales en escala de grises (modo B) de las arterias carótidas acorde a recomendaciones (34 y 35). La medición del espesor medio-intimal carotideo (EIMs) y de las placas carotideas (PC) se realizó en la pared posterior de ambas arterias carótida comúnes (derecha e izquierda), mediante ecografía en modo B con un ecógrafo Esaote Mylab 70, equipado con un transductor lineal con una frecuencia de 7-13 Mhz disponible comercialmente. Se obtuvieron imágenes de seis territorios: centímetro final de la arteria carótida común, bifurcación carotídea y primer centímetro de carótida interna en ambos lados, obteniéndose un promedio de las 3 medidas en cada lado (34-37). Se consideró presencia de placa, si se encontraba un engrosamiento irregular localizado de al menos 1,5 mm en la luz carotidea cuando 
tanto la pared arterial lejana y cercana tenían bordes afilados. Los pacientes con un EIMc <0,90 $\mathrm{mm}$ y sin placas se consideraron libres de aterosclerosis, y un EIMc $>0,90$ y / o presencia de placas se consideró aterosclerosis (38 y 39).

Se midió la VOP carótido-femoral utilizando una sonda de tonometría manual para comprimir la arteria radial derecha del sujeto. Un sistema de análisis de ondas de pulso arterial (OPA) basado en una computadora portátil (SphygmoCor, PWV Medical, Sydney, Australia) muestra un registro en tiempo real de la forma de la onda del pulso radial. Después de la adquisición de 10-12 formas de onda secuenciales, se genera una forma de onda aórtica central periférica promedio y la correspondiente derivada matemáticamente. Luego, el software VOP analiza la forma de onda central promedio para la tasa de aumento (IAC), la integral de tiempo de la presión sistólica y diastólica (ITPS / ITPD) y el tiempo de retorno de la onda reflejada (Tr) (40).

La evaluación clínica incluyó el índice de masa corporal (IMC), la evaluación de la actividad de la enfermedad mediante la puntuación de actividad de la enfermedad que incluye el recuento de 28 articulaciones (DAS28)(41), la validación Argentina del HAQ-DI (Health Assessment Questionnaire- Disability Index) (42), medición de la presión arterial y riesgo de eventos coronarios a 10 años mediante la ecuación de riesgo de Framingham 2010 (43). Todas las mediciones se realizaron al inicio del estudio y después de doce meses de tratamiento. En los pacientes que cambiaron o interrumpieron el tratamiento, las mediciones se completaron dentro del mes siguiente a la interrupción / cambio del tratamiento. 
Result ados

Desde marzo de 2012 hasta febrero de 2014 se incluyeron 64 pacientes con AR. Al ingreso al estudio y según decisión del reumatólogo tratante, 16 pacientes iniciaron tratamiento con FARME-b (Aba), 18 con FARME-b (TNFi) y 30 con FARMEc (figura 1).

Ocho pacientes completaron la primera visita y se perdieron en el seguimiento [7 con FARMEc y uno con FARME-b (Aba)]. Los 56 pacientes restantes completaron la segunda evaluación de seguimiento y fueron incluidos para el análisis final [15 en FARME-b (Aba), 18 en FARME-b (TNFi) y 23 en FARMEc] (figura 1). Cincuenta pacientes (89,3\%) eran mujeres y la edad media (DE) fue de 56,7 (12,1) años. El tiempo medio de seguimiento fue de 13,5 (DE: 7,8) años.

Las características demográficas, clínicas y de laboratorio iniciales se muestran en la tabla 1. Al inicio, los pacientes tratados con FARME-b (TNFi) tenían un EIMc significativamente más alto que los pacientes tratados con FARMEc. Los pacientes tratados con FARMEc tuvieron una duración de la enfermedad significativamente menor que los pacientes que recibieron FARME-b (TNFi) y FARME-b (Aba). Los pacientes tratados con FARME-b (TNFi) fueron más frecuentemente negativos para factor reumatoide que los pacientes tratados con FARMEc o FARME-b (Aba), mientras que la positividad para ACPA fue similar en todos los grupos. Además, los pacientes tratados con FARMEc estaban significativamente menos activos (DAS 28 basal más bajo) y tuvieron una puntuación HAQ más baja que los pacientes que recibieron FARME-b (Aba). Los pacientes en tratamiento con FARMEc, a pesar de una mayor positividad para anticuerpos, tenían niveles de actividad basales más bajos, esto podría estar directamente relacionado con estadios más tempranos de la enfermedad, debido a una menor duración de la enfermedad en este grupo. Una proporción significativamente mayor de pacientes tratados con FARME-b (Aba) (67\%), habían recibido tratamiento previo con FARME-b (TNFi), en comparación con los tratados con FARME-b (TNFi) (5\%) y FARMEc (0 \%). Al inicio del estudio, 24 pacientes (43\%) tenían aterosclerosis definida por un EIMc > 0,90 
y / o placas. No hubo diferencias en la prevalencia de aterosclerosis basal entre los diferentes grupos de tratamiento, a pesar de las mencionadas diferencias en el perfil inmunológico y la actividad de la enfermedad entre los diferentes grupos. En general, la actividad de la enfermedad mejoró durante el seguimiento, pero la diferencia solo fue significativa en el grupo FARME-b (TNFi) (tabla 3).

En el seguimiento 4 nuevos pacientes cumplieron los criterios de aterosclerosis: uno en el grupo FARME-b (Aba), dos en el grupo FARME-b (TNFi) y uno en el grupo FARMEc. Solo 2 de 56 pacientes presentaron una VOP carótido-femoral basal alta, uno en el grupo FARMEc y otro en el grupo FARME-b (Aba). No hubo diferencias significativas entre los grupos en relación al EIMc y las placas carotídeas (tabla 2). Los restantes 54/56 pacientes tenían VOP carótido-femoral basales normales y no hubo cambios significativos en ninguno de los grupos de tratamiento en el seguimiento. De manera similar, no hubo diferencias entre la línea de base y la media de seguimiento del EIMc. Dos pacientes desarrollaron nuevas placas carotídeas, uno con FARME-b (Aba) y otro con FARMEc. No hubo diferencias significativas en el desarrollo de nuevas placas entre los diferentes grupos de tratamiento. En resumen, no hubo cambios de significación estadística en los surrogantes ateroscleróticos en la segunda evaluación, en ninguno de los grupos. No hubo correlación entre la actividad de la enfermedad (DAS28) y VOP O EIMc ni al inicio ni durante el seguimiento en ninguno de los grupos de tratamiento, excepto durante el seguimiento en el grupo FARMEc. Ninguno de los pacientes desarrolló un evento cardiovascular durante el seguimiento. 
Figura 1. Diagrama de flujo de pacientes

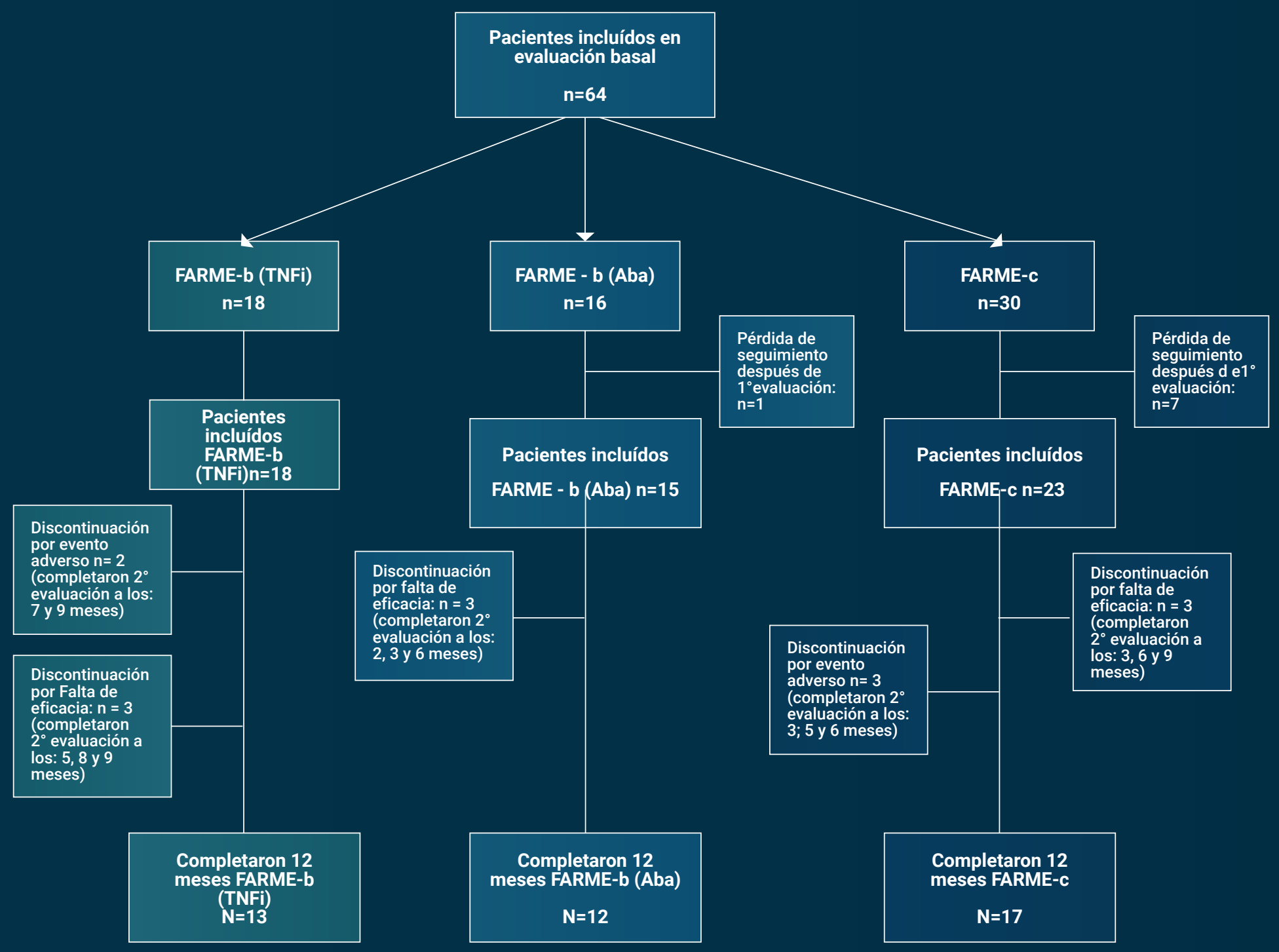

Fuente: Autores 


\section{Tabla 1. Características basales de los pacientes}

\section{por grupo de tratamiento}

\begin{tabular}{|c|c|c|c|c|c|}
\hline Características & $\begin{array}{c}\text { Todos los } \\
\text { pacientes } \\
(\mathrm{n}: 56)\end{array}$ & $\begin{array}{c}\text { FARME-b } \\
(\text { Aba) } \\
(n: 15)\end{array}$ & $\begin{array}{c}\text { FARME-b } \\
\text { (TNFi) } \\
(\mathrm{n:} 18)\end{array}$ & $\begin{array}{l}\text { FARME-c } \\
\text { (n: 23) }\end{array}$ & $\begin{array}{l}\text { Valor } \\
\text { de "p" }\end{array}$ \\
\hline Edad (años), media (DE) & $56.7(12.1)$ & $54.9(8.7)$ & $61.7(12.5)$ & $54(13)$ & 0.263 \\
\hline Femeninos, n (\%) & $50(89.3)$ & $14(93)$ & $16(89)$ & $20(87)$ & 0.823 \\
\hline $\begin{array}{l}\text { Índice de masa corporal, } \\
\text { media (DE) }\end{array}$ & $28(5.9)$ & $27(5.9)$ & $31(7)^{\star}$ & $26.1(4) \#$ & * vs \# 0.022 \\
\hline $\begin{array}{l}\text { ÍDuración de la enfermedad } \\
\text { (años), media (DE) }\end{array}$ & $11.4(10.6)$ & $13.9(10.9)+$ & $16.7(10.2)^{\star}$ & $5.5(7.7) \#$ & + vs \# $y *$ vs \# $<0.05$ \\
\hline $\begin{array}{l}\text { Factor reumatoide } \\
\text { positivo, } \mathrm{n}(\%)\end{array}$ & $45(80.3)$ & $14(93)+$ & $10(56)^{*}$ & 21 (91)\# & $+v s * y \# v s *<0.05$ \\
\hline Anti- CCP positivo, $n(\%)$ & $41(73.2)$ & $10(67)$ & $13(72)$ & $18(78)$ & 0.728 \\
\hline Tabaquistas, n (\%) & $28(50)$ & $9(60)$ & $9(50)$ & $10(44)$ & 0.609 \\
\hline Hipertesión Arterial, n (\%) & $12(21.4)$ & $3(20)$ & $6(33)$ & $3(13)$ & 0.287 \\
\hline Diabetes mellitus, $n(\%)$ & $2(3.5)$ & $0(0)$ & $1(5.6)$ & $1(4.4)$ & 0.670 \\
\hline DAS28, media (DE) & $4.7(1.3)$ & $4.8(1.1)$ & $\left.5.2(1.2)^{\star}\right)$ & $4.2(1.2) \#$ & * vs \# 0.047 \\
\hline HAQ, media (DE) & $1.16(0.8)$ & $1.5(0.8)+$ & $1.4(0.8)^{*}$ & 0.7 (0.7)\# & + vs $\# y *$ vs $\#<0.05$ \\
\hline $\begin{array}{l}\text { Colesterol total, media } \\
\text { (DE) }\end{array}$ & $199.8(51)$ & $197.1(51)$ & $202.3(44)$ & $199.8(26.2)$ & 0.9327 \\
\hline HDL, mediana (RiQ) & $55.2(12.6)$ & $54.9(9.7)$ & $57.4(13.1)$ & $53.8(14.1)$ & 0.6643 \\
\hline $\begin{array}{l}\text { Puntuación de } \\
\text { Framingham \%, (IQR) }\end{array}$ & $3.8(4.8)$ & $7.6(6.4)$ & $6.7(5.9)$ & $3.7(3.7)$ & 0.6339 \\
\hline
\end{tabular}

HAQ: Cuestionario de evaluación de la salud; DAS28: Puntaje de actividad de la enfermedad 28 Articulaciones. TNFi: Tumor Necrosis Factor inibidor. FARME: Fármacos Antirreumáticos Modificadores de la Enfermedad

\section{Fuente: Autores}


Tabla 2. Marcadores ateroscleróticos sustitutos por grupo de tratamiento, en la evaluación basal

\begin{tabular}{|l|c|c|c|c|c|}
\hline & $\begin{array}{c}\text { Todos los } \\
\text { pacientes } \\
\text { (n:56) }\end{array}$ & $\begin{array}{c}\text { FARME-b } \\
\text { (Aba) } \\
\text { (n: 15) }\end{array}$ & $\begin{array}{c}\text { FARME-b } \\
\text { (TNFi) } \\
\text { (n: 18) }\end{array}$ & $\begin{array}{c}\text { FARME-c } \\
\text { (n: 23) }\end{array}$ & $\begin{array}{c}\text { Valor } \\
\text { de “p" }\end{array}$ \\
\hline $\begin{array}{l}\text { Velocidad de onda } \\
\text { de pulso carótidofemoral } \\
\text { (VOP) (mm/s), media (DE) }\end{array}$ & $8.37(2.3)$ & $8.7(3.2)$ & $8.5(1.6)$ & $8.1(2)$ & 0.7149 \\
\hline $\begin{array}{l}\text { Espesor íntimamedia de la } \\
\text { arteria carótida común } \\
\text { (EIMC) (mm), media (DE) }\end{array}$ & $0.84(0.24)$ & $0.82(0.26)$ & $0.87(0.21)$ & $0.82(0.27)$ & 0.528 \\
\hline $\begin{array}{l}\text { Placa Carotidea } \\
\text { (PC), } n \text { (\%; IC 95\%) }\end{array}$ & $\begin{array}{l}21(37.5 ; \\
24.8-50.1) t\end{array}$ & $\begin{array}{l}5(33.3 ; 11.8- \\
62)\end{array}$ & $7(39 ; 17-64)$ & $9(39 ; 20-61)$ & 0.927 \\
\hline
\end{tabular}

TNFi: Tumor Necrosis Factor inibidor. FARME: Fármacos Antirreumáticos Modificadores de la Enfermedad 
Tabla 3. Cambio en la actividad de AR por grupo de tratamiento, en la segunda evaluación.

\begin{tabular}{|c|c|c|c|c|}
\hline FARME & $\begin{array}{c}\text { Actividad } \\
\text { de AR }\end{array}$ & Basal & $\begin{array}{l}\text { Segunda } \\
\text { evaluación } \\
\text { (12 meses) }\end{array}$ & $\begin{array}{l}\text { Valor de } \\
\text { "p" }\end{array}$ \\
\hline \multirow{3}{*}{$\begin{array}{l}\text { FARME - b } \\
\text { (Aba) } \\
(n: 15)\end{array}$} & $\begin{array}{l}\text { HAQ } \\
\text { media (DE) }\end{array}$ & $1.4(0.83)$ & $1.1(0.64)$ & 0.2156 \\
\hline & $\begin{array}{l}\text { DAS } 28 \\
\text { media (DE) }\end{array}$ & $4.9(1.1)$ & $4.6(1.2)$ & 0.3076 \\
\hline & $\begin{array}{l}\text { VSG } \\
\text { media (DE) }\end{array}$ & $42.8(28.2)$ & $40.9(28.9)$ & 0.8105 \\
\hline \multirow{3}{*}{$\begin{array}{l}\text { FARME-b } \\
\text { (TNFi) } \\
(n: 18)\end{array}$} & $\begin{array}{l}\text { HAQ } \\
\text { mean (SD) }\end{array}$ & $1.6(0.83)$ & $1.1(0.64)$ & 0.0425 \\
\hline & $\begin{array}{l}\text { DAS } 28 \\
\text { media (DE) }\end{array}$ & $5.2(1.3)$ & $3.7(1.5)$ & 0.0027 \\
\hline & $\begin{array}{l}\text { VSG } \\
\text { media (DE) }\end{array}$ & $32.8(22.3)$ & $33.2(23.1)$ & 0.9256 \\
\hline \multirow{3}{*}{$\begin{array}{l}\text { FARME-c } \\
(\mathrm{n}: 23)\end{array}$} & $\begin{array}{l}\text { HAQ } \\
\text { media (DE) }\end{array}$ & $0.55(0.51)$ & $0.75(0.63)$ & 0.0945 \\
\hline & $\begin{array}{l}\text { DAS } 28 \\
\text { media (DE) }\end{array}$ & $4.3(1.3)$ & $3.8(1.2)$ & 0.3009 \\
\hline & $\begin{array}{l}\text { VSG } \\
\text { media (DE) }\end{array}$ & 31.9 (19.3) & 30.1 (19.3) & 0.7310 \\
\hline
\end{tabular}

HAQ: Cuestionario de evaluación de la salud; DAS28: Puntaje de actividad de la enfermedad 28 Articulaciones; VSG: velocidad de sedimentación globular; TNFi: Tumor Necrosis Factor inibidor. FARME: Fármacos Antirreumáticos Modificadores de la Enfermedad. 


\section{Discusión y conclusiones}

Distintos estudios han demostrado resultados variables en la estimación del riesgo cardiovascular en pacientes con AR, utilizando la ecuación de riesgo de Framingham (44-46). Sin embargo, las nuevas herramientas de medición de riesgo, no han mejorado de manera significativa la capacidad de discriminación de la función de riesgo clásica de Framingham, ni siquiera aquellas con una magnitud de asociación a enfermedad cardiovascular (medida como odds ratio) $>3$ (47-49).

Otra limitante de nuestro trabajo es no haber discriminado los pacientes que recibían tratamiento con estatinas. Si tuvimos en consideración los niveles de colesterol al utilizar la ecuación de riesgo de Framingham.

En este estudio no encontramos cambios en los marcadores surrogados de enfermedad cardiovascular en pacientes con AR sin eventos cardiovasculares previos tratados con FARME-b (Aba), FARME-b (TNFi) o FARMEc durante 12 meses de seguimiento. Si encontramos prevalencia de aterosclerosis basal en los diferentes grupos de tratamiento, a pesar de las diferencias en el perfil inmunológico y la actividad de la enfermedad entre los diferentes grupos.

Existe cierta controversia sobre el efecto de TNFi sobre la rigidez vascular en pacientes con AR (50 y 51). Dos estudios previos han encontrado reducciones en los niveles de VOP en pacientes con AR tratados con TNFi. Wong et al, en un análisis longitudinal de datos post hoc de un ensayo aleatorizado controlado con placebo, encontraron que la VOP fue significativamente menor después de 56 semanas de tratamiento con infliximab, mientras que no hubo cambios en el EIMc ni en las placas carotídeas (51), como en nuestro estudio. La población de ese estudio fue similar a la nuestra, ya que ambos grupos excluyeron a los pacientes con eventos cardiovasculares previos. La diferencia podría estar en un seguimiento más prolongado en el trabajo de Wong. Otro pequeño estudio mostró una mejora en la VOP con etanercept, sin embargo, otros no encontraron cambios en IAC después de la terapia con TNFi (52). La VOP refleja puramente la rigidez vascular, mientras que el IAc también mide la resistencia periférica (53). De manera similar, los datos sobre el efecto de la terapia biológica sobre el EIMc son contradictorios. Del Porto et al encontraron una mejora significativa en EIMc después de 12 meses de terapia con TNFi (54), sin embargo Gonzalez Juanatey et al mostraron un empeoramiento del EIMc con este tratamiento (55). Nosotros no pudimos demostrar mejoras en el EIMc, pero es posible que el tratamiento con 
FARME, indistintamente de cual fuera, pueda prevenir el aumento del EIMc que habría ocurrido sin tratamiento. Interesantemente Wong et al también encontraron que casi la mitad de sus pacientes, en esta población seleccionada sin eventos previos y sin factores de riesgo mayores, ya tenían placas de la arteria carótida al inicio del estudio (51). En nuestro estudio, el 37\% de los pacientes incluidos tenían PC al ingreso en el estudio, lo que demuestra que estos pacientes tienen un mayor riesgo de eventos cardiovasculares. Como se ha demostrado en otros estudios, no pudimos mostrar cambios en los factores de riesgo cardiovascular modificables durante el curso del estudio (51). Sin embargo, esto podría reflejar un error de tipo II debido al pequeño tamaño del estudio.

Hay incluso menos evidencia sobre el efecto de otros productos biológicos distintos del TNFi sobre la rigidez arterial y la disfunción endotelial. Provan et al demostraron que tocilizumab y rituximab, pero no abatacept, redujeron la VOP en un pequeño grupo de pacientes con AR (24 recibieron rituximab, 5 abatacept y 7 tocilizumab) (30), el estudio evaluó a los pacientes a los 3, 6 y 12 meses de seguimiento, pero los resultados para abatacept y tocilizumab se informaron solo a los 3 meses debido al bajo número de pacientes. Mathieu et al, estudiaron la rigidez aórtica medida por VOP a los 6 meses en 21 pacientes con AR tratados con abatacept (28) y encontraron un empeoramiento de la rigidez aórtica después de 6 meses de tratamiento con abatacept, atribuido a una disminución insuficiente de la inflamación sistémica. Como en nuestro estudio, la mayoría de los pacientes tratados con abatacept habian fallado previamente al TNFi en el estudio de Mathieu. Esto refleja un sesgo de selección, dado que se seleccionaron pacientes más graves para el tratamiento con abatacept. La literatura precedente sugiere que el daño vascular aterogénico comienza antes del diagnóstico de $A R$, basado en la evidencia de un peor EIMc aterosclerótico encontrado en pacientes con AR de mayor tiempo de evolución, en comparación con AR de inicio más reciente (56 y 57).

En nuestro estudio, aunque encontramos una reducción en la actividad de la enfermedad en todos los grupos tratados, esto fue más significativo solo en los pacientes tratados con FARME-b (TNFi). Mathieu et al atribuyeron el aumento de la rigidez arterial a una disminución insuficiente de la inflamación sistémica (28). Sin embargo, nosotros no encontramos un aumento de la rigidez arterial en ninguno de los grupos terapéuticos, a pesar de una disminución similar de la inflamación sistémica.

La principal limitación de nuestro trabajo es el numero de pacientes dentro de cada grupo de tratamiento y el hecho de que no todos los pacientes completaron la evaluación a los 12 meses.

En conclusión, una gran proporción de pacientes con AR sin eventos cardiovasculares previos tenían aterosclerosis definida por EIMc y / o presencia de placas. Después de doce meses de tratamiento, no hubo cambios significativos en los marcadores surrogados de aterosclerosis en pacientes con AR de larga duración, con bajo riesgo cardiovascular basal. Los parámetros de disfunción endotelial y rigidez arterial se mantuvieron dentro de los valores normales después de un año de seguimiento cuando los pacientes fueron tratados con FARMEc, FARME-b (TNFi) o FARME-b (Aba). 
F in a n c i c ión

Este estudio fue una propuesta iniciada por un investigador, respaldada por Bristol Myers Squibb. 


\section{Referencias}

1. Pieringer $\mathrm{H}$, Pichler M. Cardiovascular morbidity and mortality in patients with rheumatoid arthritis: Vascular alterations and possible clinical implications. QJM. 2011. Jan;104(1):13-26. doi: 10.1093/qjmed/hcq203. Epub 2010 Nov 10. PMID: 21068083.

2. Meune C, Touzé E, Trinquart L, Allanore Y. Trends in cardiovascular mortality in patients with rheumatoid arthritis over 50 years: a systematic review and meta-analysis of cohort studies. Rheumatology (Oxford). 2009;48(10):1309-13.

3. Gabriel SE. Cardiovascular Morbidity and Mortality in Rheumatoid Arthritis. Am J Med. 2008 Oct;121(10 Suppl 1):S9-14. doi: 10.1016/j.amjmed.2008.06.011. PMID: 18926169; PMCID: PMC2858687.

4. England BR, Thiele GM, Anderson DR, Mikuls TR. Increased cardiovascular risk in rheumatoid arthritis: mechanisms and implications. BMJ. 2018 Apr 23;361:k1036. doi: 10.1136/bmj.k1036. PMID: 29685876; PMCID: PMC6889899.

5. DeMizio DJ, Geraldino-Pardilla LB. Autoimmunity and Inflammation Link to Cardiovascular Disease Risk in Rheumatoid Arthritis. Rheumatol Ther 7, 19-33 (2020). https://doi.org/10.1007/s40744-019-00189-0

6. Park YB, Ahn CW, Choi HK, Lee SH, In BH, Lee $\mathrm{HC}$, et al. Atherosclerosis in rheumatoid arthritis:
Morphologic evidence obtained by carotid ultrasound. Arthritis Rheum. 2002;46(7):1714-9.

7. Kumeda $Y$, Inaba M, Goto H, Nagata M, Henmi $Y$, Furumitsu $Y$, et al. Increased thickness of the arterial intima-media detected by ultrasonography in patients with rheumatoid arthritis. Arthritis Rheum. 2002 Jun;46(6):1489-97. doi: 10.1002/art.10269. PMID: 12115178.

8. Del Rincón I, Freeman GL, Haas RW, O'Leary $\mathrm{DH}$, Escalante A. Relative contribution of cardiovascular risk factors and rheumatoid arthritis clinical manifestations to atherosclerosis. Arthritis Rheum. 2005;52(11):3413-23.

9. Del Rincón I, Williams K, Stern MP, Freeman $\mathrm{GL}$, Escalante A. High incidence of cardiovascular events in a rheumatoid arthritis cohort not explained by traditional cardiac risk factors. Arthritis Rheum. 2001;44(12):2737-45.

10. Jagpal A, Navarro-Millán I. Cardiovascular co-morbidity in patients with rheumatoid arthritis: A narrative review of risk factors, cardiovascular risk assessment and treatment. BMC Rheumatol. 2018;2(1):1-14.

11. Wei T, Yang B, Liu H, Xin F, Fu L. Development and validation of a nomogram to predict coronary heart disease in patients with rheumatoid arthritis in northern China. Aging (Albany NY). 2020;12(4):3190-204. 
12. Laurent S, Boutouyrie P. Arterial stiffness: A new surrogate end point for cardiovascular disease? J Nephrol. 2007 Nov-Dec;20 Suppl 12:S45-50. PMID: 18050143.

13. Roman MJ, Devereux RB, Schwartz JE, Lockshin MD, Paget SA, Davis A, et al. Arterial stiffness in chronic inflammatory diseases. Hypertension. 2005;46(1):194-9.

14. Cohn JN. Arterial stiffness, vascular disease, and risk of cardiovascular events. Circulation. 2006;113(5):601-3.

15. Narkiewicz K, Kjeldsen SE, Oparil S, Hedner T. Hypertension and cardiovascular disease: Is arterial stiffness the heart of the matter. Blood Press. 2007;16(4):236-7.

16. Kim ED, Ballew SH, Tanaka H, Heiss G, Coresh J, Matsushita K. Short-term prognostic impact of arterial stiffness in older adults without prevalent cardiovascular disease. Hypertension. 2019 Dec;74(6):1373-1382. doi: 10.1161/HYPERTENSIONAHA.119.13496. Epub 2019 Nov 4. PMID: 31679417; PMCID: PMC7110414.

17. Shahzad R, Shankar A, Amier R, Nijveldt R, Westenberg JJM, De Roos A, et al. Quantification of aortic pulse wave velocity from a population based cohort: A fully automatic method. J Cardiovasc Magn Reson. 2019;21(1):1-14.

18. Ferrari AU, Radaelli A, Centola M. Physiology of Aging Invited Review: Aging and the cardiovascular system. J Appl Physiol. 2003;95:2591-7.

19. Safar ME, Henry O, Meaume S. Aortic pulse wave velocity: an independent marker of cardiovascular risk. Am J Geriatr Cardiol. 2002 Sep-Oct;11(5):295-8.

doi: 10.1111/j.1076-7460.2002.00695.x.

PMID: 12214167.

20. Mattace-Raso FUS, Hofman A, Verwoert GC, Wittemana JCM, Wilkinson I, Cockcroft J, et al. Determinants of pulse wave velocity in healthy people and in the presence of cardiovascular risk factors: 'Establishing normal and reference values'. Eur Heart J. 2010;31(19):2338-50.

21. Blacher J, Guerin AP, Pannier B, Marchais SJ, Safar ME, London GM. Impact of aortic stiffness on survival in end-stage renal disease. Circulation. 1999 May 11;99(18):2434-9. doi: 10.1161/01.cir.99.18.2434. PMID: 10318666.

22. Choy E, Ganeshalingam K, Semb AG, Szekanecz Z, Nurmohamed M. Cardiovascular risk in rheumatoid arthritis: Recent advances in the understanding of the pivotal role of inflammation, risk predictors and the impact of treatment. Rheumatol (United Kingdom). 2014;53(12):2143-54.

23. Chighizola C, Schioppo T, Ingegnoli F, Luigi Meroni P. Potential Effect of Anti-Inflammatory Treatment on Reducing the Cardiovascular Risk in Rheumatoid Arthritis. Curr Vasc Pharmacol. 2012 Sep;10(5):639-46. doi: 10.2174/157016112801784503. PMID: 22272912.

24. Barbhaiya M, Solomon DH. Rheumatoid arthritis and cardiovascular disease: An update on treatment issues. Curr Opin Rheumatol. 2013 May;25(3):317-24.

doi: 10.1097/BOR.0b013e32835fd7f8. PMID: 23466960; PMCID: PMC4836607

25. Tam HW, Chen CM, Leong PY, Chen CH, Li YC, Wang $\mathrm{YH}$, et al. Methotrexate might reduce ischemic 
stroke in patients with rheumatoid arthritis: a population-based retrospective cohort study. Int J Rheum Dis. 2018 Aug;21(8):1591-1599. doi: 10.1111/1756-185X.13267. Epub 2018 Jan 25. PMID: 29372595.

26. Dhillon N, Liang K. Prevention of Stroke in Rheumatoid Arthritis. Curr Treat Options Neurol. $2015 \quad J u l ; 17(7): 356 . \quad$ doi: 10.1007/s11940-015-0356-3. PMID: 25981388.

27. Reiss AB, Silverman A, Khalfan M, Vernice NA, Kasselman LJ, Carsons SE, et al. Accelerated Atherosclerosis in Rheumatoid Arthritis: Mechanisms and Treatment. Curr Pharm Des. 2019;25(9):969-986. doi: 10.2174/1381612825666190430113212. PMID: 31208307.

28. Mathieu S, Couderc M, Glace B, Pereira B, Tournadre A, Dubost JJ, et al. Effects of 6 months of abatacept treatment on aortic stiffness in patients with rheumatoid arthritis. Biol Targets Ther. 2 de diciembre de 2013;7(1):259-64.

29. Virone A, Bastard JP, Fellahi S, Capeau J, Rouanet S, Sibilia J, et al. Comparative effect of tumour necrosis factor inhibitors versus other biological agents on cardiovascular risk-associated biomarkers in patients with rheumatoid arthritis. RMD Open. 2019 Jul 21;5(2):e000897. doi: 10.1136/rmdopen-2019-000897. PMID: 31413865; PMCID: PMC6667971.

30. Provan SA, Berg IJ, Hammer HB, Mathiessen A, Kvien TK, Semb AG. The impact of newer biological disease modifying anti-rheumatic drugs on cardiovascular risk factors: A 12-month longitudinal study in rheumatoid arthritis patients treated with rituximab, abatacept and tociliziumab. PLoS One. 2015 Jun 26;10(6):e0130709. doi: 10.1371/journal.pone.0130709. PMID: 26114946; PMCID: PMC4482693.

31. Aletaha D, Neogi T, Silman AJ, Funovits J, Felson DT, Bingham CO, et al. 2010 Rheumatoid arthritis classification criteria: An American College of Rheumatology/European League Against Rheumatism collaborative initiative. Ann Rheum Dis. 2010;69(9):1580-8.

32. Aletaha D, Neogi T, Silman AJ, Funovits J, Felson DT, Bingham CO, et al. 2010 Rheumatoid arthritis classification criteria: An American College of Rheumatology/European League Against Rheumatism collaborative initiative. Arthritis Rheum. 2010;62(9):2569-81.

33. Scott DL, Wolfe F, Huizinga TWJ. Rheumatoid arthritis. Lancet. 2010 Sep 25;376(9746):1094-108. doi: $\quad 10.1016 / S 0140-6736(10) 60826-4$. PMID: 20870100.

34. Hoeks APG, Willekes C, Boutouyrie P, Brands PJ, Willigers JM, Reneman RS. Automated detection of local artery wall thickness based on M-line signal processing. Ultrasound Med Biol. 1997;23(7):1017-23.

35. Naredo E, Möller I, Gutiérrez M, Bong DA, Cobo T, Corominas $\mathrm{H}$, et al. Multi-examiner reliability of automated radio frequency-based ultrasound measurements of common carotid intima-media thickness in rheumatoid arthritis. Rheumatology. 2011;50(10):1860-4.

36. Corrales A, González-Juanatey C, Peiró ME, Blanco R, Llorca J, González-Gay MA. Carotid ultrasound is useful for the cardiovascular risk stratification of patients with rheumatoid arthritis: Results of a population-based study. Ann Rheum Dis. $2014 \quad$ Apr;73(4):722-7. doi: 
10.1136/annrheumdis-2012-203101. Epub 2013 Mar 16. PMID: 23505241.

37. Touboul PJ, Hennerici MG, Meairs S, Adams $\mathrm{H}$, Amarenco P, Bornstein N, et al. Mannheim carotid intima-media thickness consensus (2004-2006): An update on behalf of the advisory board of the 3rd and 4th Watching the Risk Symposium 13th and 15th European Stroke Conferences, Mannheim, Germany, 2004, and Brussels, Belgium, 2006. Cerebrovasc Dis. 2007;23(1):75-80. doi: 10.1159/000097034. Epub 2006 Nov 14. PMID: 17108679 .

38. Gonzalez-Juanatey C, Llorca J, Martin J, Gonzalez-Gay MA. Carotid Intima-Media Thickness Predicts the Development of Cardiovascular Events in Patients with Rheumatoid Arthritis. Semin Arthritis Rheum. 2009 Apr;38(5):366-71. doi: 10.1016/j.semarthrit.2008.01.012. Epub 2008 Mar 12. PMID: 18336869.

39. Evans MR, Escalante A, Battafarano DF, Freeman GL, O'Leary DH, Del Rincõn I. Carotid atherosclerosis predicts incident acute coronary syndromes in rheumatoid arthritis. Arthritis Rheum. 2011 May;63(5):1211-20. doi: 10.1002/art.30265. PMID: 21305526; PMCID: PMC3286362.

40. Klocke R, Cockcroft JR, Taylor GJ, Hall IR, Blake DR. Arterial stiffness and central blood pressure, as determined by pulse wave analysis, in rheumatoid arthritis. Ann Rheum Dis. 2003 May;62(5):414-8. doi: 10.1136/ard.62.5.414. PMID: 12695151; PMCID: PMC1754549.

41. Prevoo MLL, Van'T Hof MA, Kuper HH, Van Leeuwen MA, Van De Putte LBA, Van Riel PLCM. Modified disease activity scores that include twenty-eight-joint counts development and validation in a prospective longitudinal study of patients with rheumatoid arthritis. Arthritis Rheum. 1995;38(1):44-8.

42. Citera G, Arriola MS, Maldonado-Cocco JA, Rosemffet MG, Sánchez MM, Goñi MA, et al. Validation and crosscultural adaptation of an Argentine Spanish version of the Health Assessment Questionnaire disability index. J Clin Rheumatol. 2004;10(3):110-5.

43. D’Agostino RB, Vasan RS, Pencina MJ, Wolf PA, Cobain M, Massaro JM, et al. General cardiovascular risk profile for use in primary care: The Framingham heart study. Circulation. 2008;117(6):743-53.

44. Jafri K, Ogdie A, Qasim A, Patterson SL, Gianfrancesco $M$, Izadi Z, et al. Discordance of the Framingham cardiovascular risk score and the 2013 American College of Cardiology/American Heart Association risk score in systemic lupus erythematosus and rheumatoid arthritis. Clin Rheumatol [Internet]. 9 de febrero de 2018;37(2):467-74. Disponible en: http://link.springer.com/10.1007/s10067-017-3860$x$

45. Dessein PH, Corrales A, Lopez-Mejias R, Solomon A, Woodiwiss AJ, Llorca J, et al. The framingham score and the systematic coronary risk evaluation at low cutoff values are useful surrogate markers of high-risk subclinical atherosclerosis in patients with rheumatoid arthritis. J Rheumatol. 2016;43(3):486-94.

46. Korkmaz M, Karaaslan F, Erdogan Y, Bolat E, Karacavus S, Kizilkaya H, et al. Efficacy of treatment with glucosamine sulfate in patients with knee effusion due to osteoarthritis. Pakistan J Med Sci. 2013;29(3):847-50. 
47. Lippi G, Salvagno GL, Targher G, Guidi GC. Multiple biomarkers for the prediction of first major cardiovascular events and death: Considerable costs and limited benefits. MedGenMed Medscape Gen Med. 2007;9(1):2631-9.

48. Lloyd-Jones DM, Liu K, Tian L, Greenland P. Narrative review: Assessment of C-reactive protein in risk prediction for cardiovascular disease. Ann Intern Med. 2006;145(1):35-42.

49. Ware JH. The Limitations of Risk Factors as Prognostic Tools. N Engl J Med. 2006;355(25):2615-7.

50. Kume K, Amano K, Yamada S, Hatta K, Ohta H, Kuwaba N. Tocilizumab monotherapy reduces arterial stiffness as effectively as etanercept or adalimumab monotherapy in rheumatoid arthritis: An open-label randomized controlled trial. J Rheumatol. 2011;38(10):2169-71.

51. Wong $M$, Oakley SP, Young $L$, Jiang BY, Wierzbicki A, Panayi G, et al. Infliximab improves vascular stiffness in patients with rheumatoid arthritis. Ann Rheum Dis. 2009;68(8):1277-84.

52. Mäki-Petäjä KM, Hall FC, Booth AD, Wallace SML, Yasmin, Bearcroft PWP, et al. Rheumatoid arthritis is associated with increased aortic pulse-wave velocity, which is reduced by anti-tumor necrosis factor-alpha therapy. Circulation. 2006 Sep 12;114(11):1185-92. doi: 10.1161/CIRCULATIONAHA.105.601641. Epub 2006 Sep 4. PMID: 16952987.

53. Sakurai M, Yamakado T, Kurachi H, Kato T, Kuroda K, Ishisu R, et al. The relationship between aortic augmentation index and pulse wave velocity: An invasive study. J Hypertens. 2007;25(2):391-7.
54. Del Porto F, Laganà B, Lai S, Nofroni I, Tinti F, Vitale $\mathrm{M}$, et al. Response to anti-tumour necrosis factor alpha blockade is associated with reduction of carotid intima-media thickness in patients with active rheumatoid arthritis. Rheumatology. 2007;46(7):1111-5.

55. Gonzalez-Juanatey C, Llorca J, Garcia-Porrua C, Martin J, Gonzalez-Gay MA. Effect of anti-tumor necrosis factor a therapy on the progression of subclinical atherosclerosis in severe rheumatoid arthritis. Arthritis Care Res. 2006;55(1):150-3.

56. del Rincón I, O'Leary DH, Freeman GL, Escalante A. Acceleration of atherosclerosis during the course of rheumatoid arthritis. Atherosclerosis. 2007 Dec;195(2):354-60. doi: 10.1016/j.atherosclerosis.2006.09.027. Epub 2006 Nov 13. PMID: 17097659.

57. Hannawi S, Haluska B, Marwick TH, Thomas R. Atherosclerotic disease is increased in recent-onset rheumatoid arthritis: A critical role for inflammation. Arthritis Res Ther. 2007;9(6):R116. doi: 10.1186/ar2323. PMID: 17986352; PMCID: PMC2246234. 
GLOBAL RHEUMATOLOGY 